DOI: http://dx.doi.org/10.33846/hn40402

http://heanoti.com/index.php/hn

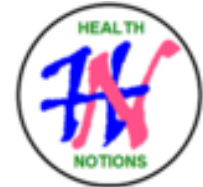

RESEARCH ARTICLE

URL of this article: http://heanoti.com/index.php/hn/article/view/hn40402

\title{
Use of The Term Resilience in Adult Cancer Patients: A Systematic Review of A Qualitative Study
}

\author{
La Rakhmat Wabula ${ }^{1(\mathrm{CA})}$, Syahfitrah Umamity ${ }^{2}$, Ellen Lombonaung ${ }^{3}$ \\ ${ }^{1(\mathrm{CA})}$ Departemen of Medical-Surgical Nursing, Nursing Study Programme, STIKes Maluku Husada, Indonesia; \\ la.rakhmat.wabula.stikesmh@gmail.com (Corresponding Author) \\ ${ }^{2}$ Department of Maternity Nursing, Nursing Study Programme, STIKes Maluku Husada, Indonesia; \\ syahfitrah.umamity@gmail.com \\ ${ }^{3}$ Departmentof Community Nursing, Nursing Study Programme, STIKes Maluku Husada, Indonesia; \\ ellenpattinasarany1970@gmail.com
}

\begin{abstract}
Resilience was a dynamic process that adapts positively to the difficulties it has. This term was often used by researchers in cancer patients. Controversy often occurs in its conception and little is known by patients in the use of the term resilience in their lives. The subject of this paper was cancer patients. The literature review was conducted by examining qualitative studies that discuss the use of terms in cancer patients and the use of themes used in terms of resilience. A systematic review of reviews through SCOPUS, MEDLINE, Psyc INFO and CINAHL reviewed from 2000 to 2017. Thematic analysis was used to encode themes in the studio and produce analytical themes, and resolution resolutions in the studio. After being invited to 573 citations, there were only 32 suitable studies. Four categories emerged, including coping strategies, social support, spirituality, and growth. The conclusion is no use of the term resilience in cancer patients, but cancer patients more often use other terms with the same meaning as resilience. Cancer patients rarely use the term direct resilience, but more often use terms such as coping strategies, social support, growth, and spiritual as terms related to resilience.
\end{abstract}

Keywords: resilience; cancer patients

\section{INTRODUCTION}

\section{Background}

Resilience, a dynamic process of positive adaptation within the context of significant adversity ${ }^{(1)}$, has been linked to various positive health outcomes, including reduced psychological distress, protection from depression, and improved quality of life ${ }^{(2,3)}$. However, despite the vast body of literature regarding resilience, significant controversy exists about its conceptualization, and there is a lack of consistency regarding how the term resilience is used and defined ${ }^{(1)}$.

While some researchers use the term resilience to describe pre-existing personality traits, others regard it as a dynamic process of adaptation that develops over time, while a third perspective argues for resilience as a psychosocial outcome ${ }^{(1,4)}$. An integrated biopsychosocial approach to resilience harmonizes all these prior definitions by concept of resilience as the psychological equivalent of a somatic immune system, protecting against adversity through multi-level defence mechanisms ${ }^{(5)}$. Thus, while some resilience mechanisms may be innate/pre-existing, others may be developed through individual adaptation, or through external influences. Furthermore, when exposed to adversity, resilience may grow in effectiveness through Behavioral immunization, allowing a more effective response to that specific adversity in the future ${ }^{(6)}$.

The concept of resilience has attracted considerable interest in cancer. A diagnosis of cancer represents substantial adversity and is often associated with significant physical and emotional distress, at times resulting in mental health conditions such as depression and anxiety ${ }^{(1)}$. Resilience promoting interventions would seem 
advantageous for cancer patients but at present, evidence for such interventions is limited in the cancer setting, with individual results ranging from no statistically significant change in quality of life, to reduced distress and improved quality of life ${ }^{(5)}$. One reason for this may be a lack of consistency in how the term resilience is used and defined within the oncology setting.

To date, there have been two reviews of resilience in adult cancer care. Eicher and colleagues undertook a review of 11 quantitative papers with the aim of describing current scientific perspectives of resilience in adult cancer care and their implications for cancer nursing ${ }^{(3)}$. They defined resilience as a dynamic process of facing adversity related to cancer and confirmed the association of resilience with improved health outcomes, recommending the development of a conceptual framework for nursing interventions and the refinement of scales/instruments to measure resilience ${ }^{(3)}$.

Molina and colleagues reviewed 57 papers to describe the ways in which elements of resilience have been defined and studied at each phase of the cancer continuum from risk assessment/screening to survivorship and end of life. They concluded that as the stress and adaptation required at each phase may be different, each phase of the cancer experience has unique as well as shared aspects of resilience. In all phases of the cancer continuum, resilience attributes included baseline characteristics, mechanisms of adaptation and psychosocial outcomes $^{(6)}$. In the limitations of their reviews, Molina acknowledged the diversity of definitions, literature, and study design, while Eicher acknowledged the exclusion of qualitative studies on resilience.

\section{Purpose}

Reviewing qualitative literature that focuses on patient usage of the term resilience may add to the conceptual definitions of resilience, but to date, no systematic literature review has synthesis the qualitative research in adult cancer care examining how patients use the term resilience in their day-today life. To address this gap, the aim of the present review was to enhance the understanding of cancer patients' use of resilience with a focus on the following questions: (1) what are the definitions of resilience as identified by patients and researchers/study authors? and (2) what are the themes relating to attributes of resilience as identified by patients?

\section{METHODS}

This review followed guidelines from the Enhancing Transparency in Reporting the Synthesis of Qualitative Research (ENTREQ) statement ${ }^{(7)}$.

\section{Inclusion and Exclusion Criteria}

To meet inclusion criteria, studies needed to have a qualitative design, be printed in English and include patients or survivors over the age of 18 with any type or stage of cancer. Qualitative studies were defined as using qualitative methods of data collection, such as interviews and focus groups and using qualitative data analysis methods such as thematic analysis or discourse analysis. Studies needed to include "resilience" and "cancer" (or a derivative) in their title, abstract, or keywords and in full text. Studies were excluded if resilience was not a theme or outcome, was studied primarily from the perspective of non-patient members (family members or caregivers), or was defined in the context of a group rather than an individual (i.e. family resilience).

\section{Search Strategies}

A pre-planned comprehensive search of four electronic databases (SCOPUS, MEDLINE, PsycINFO, and CINAHL) was conducted to identify all relevant studies between database inception and the end of November 2017. The search strategy (initially created in MEDLINE then accurately translated for the other databases) involved using database-specific controlled terms (where available) and the key words "resilience" and "cancer" (or derivatives such as melanoma), as well as Boolean operators in the title and abstract and medical subject headings. Qualitative studies were identified by using the keyword "qualitative" and searching for studies using qualitative methodology, such as phenomenology or qualitative analysis methods. A single author screened study titles and abstracts to identify studies for full-text review. An example of the search strategies used is provided in Figure 1. 


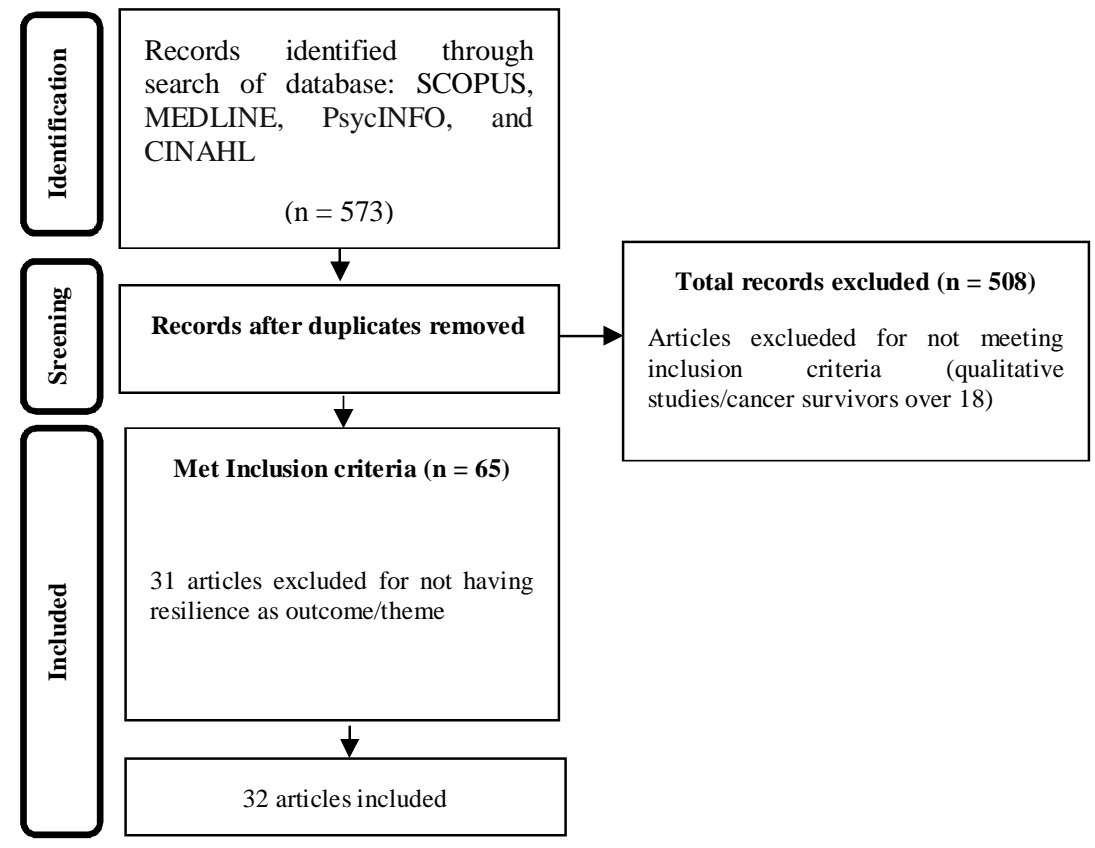

Figure 1. Diagram summarizing search of literature

\section{RESULTS}

\section{Definitions of Resilience}

No patient definitions of resilience were identified within the reviewed studies, and patient usage of the term resilience was only reported in a single study ${ }^{(8)}$. Resilience was explicitly defined eight times by researchers in the included studies with varying definitions as shown in Table 1. Four definitions described a process of adaptation to adversity or threats ${ }^{(9)}$, with one study explicitly noting the adaptation is positive ${ }^{(10)}$. Two definitions described resilience as a phenomenon of maintained or recovered psychological health after adversity $^{(10,11)}$, with one study adding the concept of physical changes, and suggested the notion of "growing past"(10). One study defined resilience as "a comprehensive process, including existential meaning making, selection and optimisation (of goals) and growth" ${ }^{(8)}$, and the final study defined resilience as having three main components: social embeddedness, positive life perspective, and personal resourcefulness ${ }^{(12)}$.

\section{Themes of Resilience}

Figure 2 depicts the conceptual map of themes identified in the thematic analysis, and a full list of themes can be found in the supplementary material. Seventy-nine unique themes of resilience were initially identified as used by authors of individual studies, which were then combined based on similarity. Studies contributed between one and four individual themes, with each study contributing a mean of three individual themes. Four overarching thematic categories emerged and were ordered according to prevalence: (1) coping, (2) social support, (3) spirituality, and (4) growth. Fourteen studies identified resilience themes within only one category, 15 studies identified themes within two categories, two studies identified resilience themes across three main categories, and one studyidentified themes across all four categories. Spirituality, growth, and social support were commonly grouped with coping strategies due to the plethora of coping strategies raised as a theme, and spirituality was associated with coping in all but one study ${ }^{(8)}$. No other clusters of themes were evident within the analysed studies. 


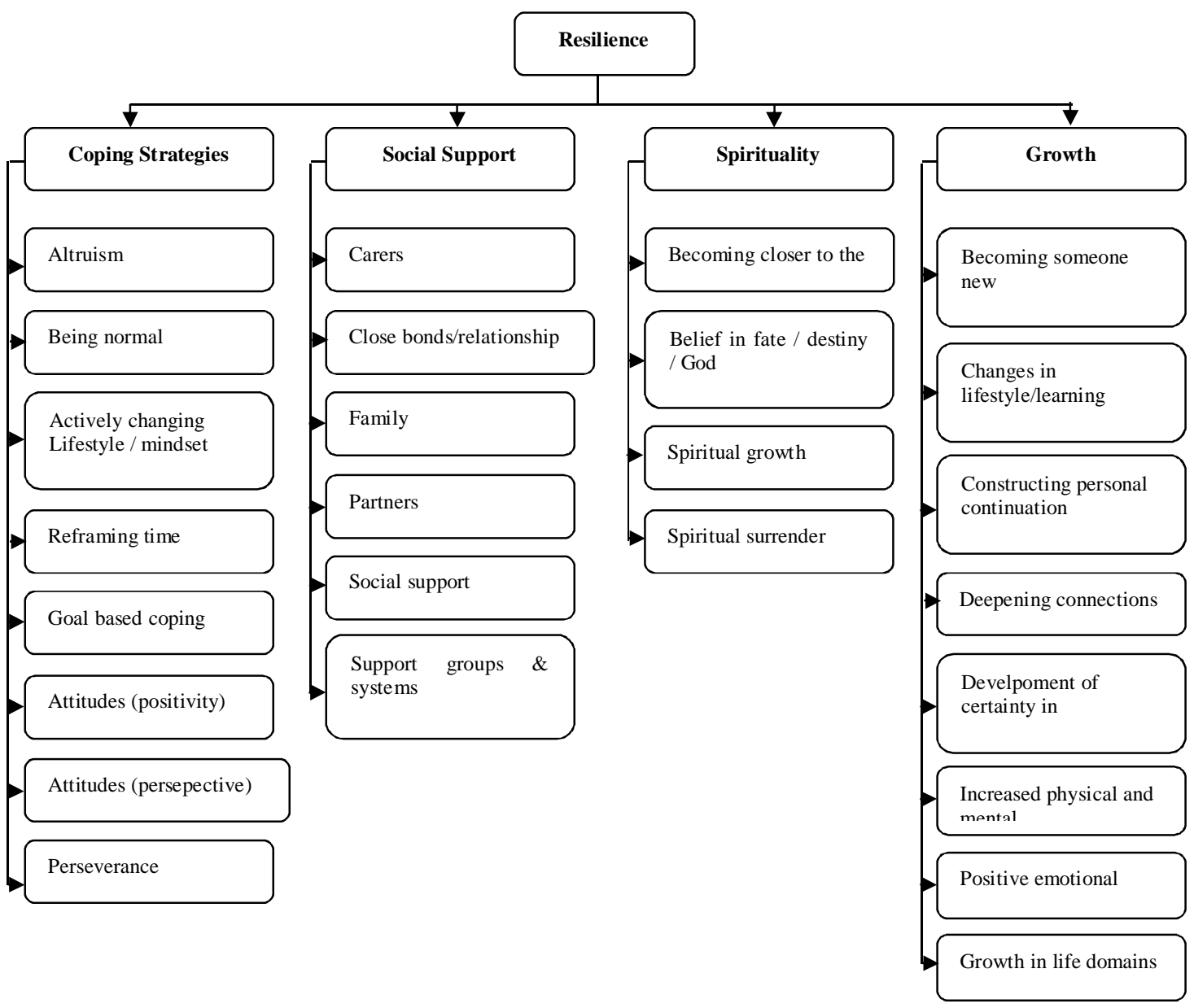

Figure 2. Conceptual map of themes of resillience

Coping

The thematic category most commonly associated with resilience was coping with the stressors of the cancer experience, which was identified in all but one study ${ }^{(13)}$. Themes related to coping emerged 55 times across all studies and were further summarized under the following eight subordinate themes: positivity, perspectives, perseverance, being normal/denial, actively changing lifestyle and mindset, reframing time, altruism, and goal-based coping, summarized in detail below.

Maintaining a positive attitude was a frequently discussed coping strategy, succinctly expressed in one study as "if I was a negative person, I wouldn't be sitting here with you today". Variations of positivity as a theme of resilience included thinking positively and remaining positive ${ }^{(14)}$, having the right mindset ${ }^{(15)}$, being grateful $^{(10)}$, and seeing the bright side $^{(2,10,14,15)}$. The perspectives of the patient helped shape their coping strategies, with studies describing this theme as acceptance of their diagnosis, being self-reliant ${ }^{(9)}$, or identifying the meaning of life and the situation ${ }^{(12)}$. Being pragmatic and seeing things in perspective were variants of this theme ${ }^{(14,16)}$.

The concept of persevering through cancer-related stress is another coping strategy patients used frequently; one study identified this theme as "doing what has to be done"(9), and a patient recounts her experience of perseverance as "these are challenging times and may be rough times, but keep on" (26). Others described this theme as inner strength ${ }^{(17)}$, not giving up ${ }^{(10)}$ or stoicism ${ }^{(18)}$. Perseverance was described in one study as the process of handling decline and loss, where patients endure tough treatment periods to have a life prolonging effect ${ }^{(9)}$. In contrast, the concept of "toxic" resilience was raised, where the patient's drive to stay resilient eventually becomes problematic and counterproductive to quality of life in the participants ${ }^{(18)}$.

Another coping strategy displayed was the concept of being "normal", with one patient stating "I think what I wanted to preserve was the absolute preciousness of normality" (19). This was achieved through methods such as minimizing the impact of cancer ${ }^{(20)}$, avoiding conventional sick roles ${ }^{(14)}$, maintaining normal life ${ }^{(9),}$ and 
coping through distractions, such as work ${ }^{(5,12)}$. Some patients sought to cope by actively changing one's mindset and lifestyle, with variations of this theme including being more flexible ${ }^{(16)}$, dealing with adversity ${ }^{(17)}$, embracing paradoxes ${ }^{(21)}$, existential meaning making through selection and optimization of goals ${ }^{(8)}$, finding ways to keep going ${ }^{(21)}$, and having a voice in treatment ${ }^{(13)}$. Reframing time was another common mechanism of coping ${ }^{(21)}$, with another related strategy being living in the present ${ }^{(21)}$. Other coping strategies include helping others/altruism $^{(22)}$, assimilation or accommodation of cancer through goal-based coping ${ }^{(18,19)}$, and surrendering to life impermanence and circumstances ${ }^{(23)}$.

\section{Social support}

Resilience was commonly described in a background of receiving support from friends, family, community, and Health care workers and was described 10 times as a theme of resilience. A study conducted in the USA on 13 older adult patients with lung, prostate, or digestive system cancers stated that social support contributed to resilience and held much symbolic and literal meaning for patients, suggesting that this was one aspect of resilience which allowed them to maintain and grow past their previous level of functioning. Conversely, participants characterized as non-resilient were described as remarkable in their lack of social support ${ }^{(10)}$. Similarly, another patient described the support her family provided as "My family never left me alone ... if it was not for them, I do not know what I would have done"(5). The importance of supportive relationships was further shown in another study when women resilient to distress had stable, supportive families, while the sole participant with a lack of family support displayed distress ${ }^{(24)}$. Other social supports discussed included partners and ex-partners ${ }^{(3,25)}$, children, friends, religious, and social groups and healthcare professionals $^{(17)}$.

Growth

Growth, described as growth in one or more life domains following exposure to cancer-related stressors, was raised as a theme of resilience nine times across all identified studies. Patients resilient to distress often demonstrated growth in at least one domain including changes in worldview, faith, and family relations ${ }^{(9)}$. Growth as a theme was identified as becoming someone new, with new values, causing the patients to become more resilient ${ }^{(20)}$. One study described the process as the deepening of connections with others and spiritual forces, stating cancer "prompted everyone to connect with someone bigger", ${ }^{, 23)}$. Another study discussed the development and growth of personal strength as a consequence of cancer, one patient stating "I don't think there's any way I'd be so positive or determined. I think that totally came from that experience"(26). Growth was not limited to cancerrelated growth; mental/physical growth and improved self-esteem occurred in patients with cancer who practiced yoga ${ }^{(13)}$.

\section{Spirituality}

Spirituality and religion were a theme of resilience in several of the studies, occurring nine times across all identified studies as a theme of resilience, with eight unique themes. The idea of entrusting one's self to fate and destiny was expressed by one patient who stated "I believe nothing can happen without His (God) will ... my prayers helped me and gave me strength"(22). Other patients discussed cancer being an opportunity to become closer to the Lord and emphasized the importance of religion to their coping with cancer ${ }^{(15)}$. Spirituality was characterized in one study in the context of a "belief frame" ranging from traditional Christian faith to atheism, which participants experienced as something positive and contributory to resilience ${ }^{(8)}$. Spirituality-faith was similarly described in another study as expressing feelings of gratitude and being blessed; patients who demonstrated spirituality were characterized by a general lack of fear ${ }^{(10)}$. A comparable concept was characterized as "spiritual surrender" a form of spiritual awakening which enables patients to find inner peace and comfort by surrendering to impermanence ${ }^{(27)}$.

\section{DISCUSSION}

This systematic literature review aimed to determine the definitions of resilience as identified by researchers/study authors in examining patient experiences of resilience and to determine the themes relating to attributes of resilience as identified by patients. Our review showed that use of the term resilience by patients was rare; no studies included a patient definition of resilience, and only one study ${ }^{(9)}$ included a patient use of the term resilience. Whether this is because patients did not use the word or researchers did not include patient usage of the term is unclear. However, none of the studies included questions on what the term resilience meant to subjects; in fact, one of the included studies ${ }^{(10)}$ explicitly stated that the usage of the term resilience was actively avoided by researchers while formulating questions and the term was never brought up during 
interviews. Researcher definitions of resilience were lacking in the 32 studies included in the review; only eight studies provided an explicit in-text definition of the term resilience. The most common definition to emerge from the included studies described a process of adaptation in response to threats or adversity; however, the definitions used varied widely, supporting the idea that resilience is poorly defined and potentially poorly understood and may be one of the factors contributing to the varying effects of resilience as reported in extant literature.

Although the term resilience was not commonly used by either researchers or patients, coping strategies, social support, growth, and spirituality were the most commonly identified attributes of resilience. Coping strategies were the most common theme, being represented in nearly every study, and appeared as a stand-alone theme in more than half of the studies. Coping strategies included concepts such as positivity, perspectives, perseverance, being normal/denial, actively changing lifestyle, and mindset, reframing time, altruism, and goalbased coping. Social support was the next most common theme and involved individuals receiving support from external sources, such as friends, family, healthcare workers, and cancer support groups. Growth was another theme of resilience, with individuals exhibiting growth in various life domains, and spirituality emerged as the final theme, with the beliefs of individuals contributing towards resilience. Coping strategies were the main theme most commonly grouped with other main themes, and spirituality was grouped with coping in all but one study ${ }^{(28)}$, suggesting that spirituality may act as a coping mechanism as well as a main theme. Additionally, only psychosocial aspects of resilience were identified as themes of resilience, suggesting that other aspects of resilience such as physical resilience were either not discussed by patients, or not reported by researchers.

One finding of interest was how growth was conceptualized as a theme of resilience; although growth has been included in some definitions of resilience ${ }^{(10)}$, most definitions refer to a return to baseline functioning ${ }^{(10)}$. This finding suggests that researchers may use the term resilience interchangeably with posttraumatic growth. Although a similar construct to resilience, posttraumatic growth is described as "positive psychological change experienced as a result of the struggle with highly challenging life circumstances", implying individuals achieve a higher level of functioning than before the trauma ${ }^{(29)}$ and has been both positively and negatively associated with resilience ${ }^{(4)}$. While this does not discount growth as a theme of resilience, further research should be undertaken to investigate whether resilience is conflated with posttraumatic growth. It is unclear from the analysis of the studies whether the attributes of resilience were used to describe resilience as a process of adaptation, an innate trait associated with resilient behaviors or an outcome. Many of the individuals who appeared resilient displayed similar attributes such as positive or stoic attitudes and had good social support; similarly, Lam et al. and Pentz ${ }^{(10,12)}$ described individuals lacking in resilience as lacking social support, suggesting that resilience has characteristics of a trait and a process facilitated by personal factors and resources, such as social support. As this review was unable to determine whether resilience is best characterized as a trait, process, outcome, or a combination of these descriptions, a longitudinal approach may be more appropriate to examine how resilience changes over time and thus clarify its construct definition.

The current review has notable limitations. As many of the identified articles did not focus on resilience as a primary goal of analysis, patient usage and definitions of the term may not have arisen during the course of each study, or may have been omitted. Similarly, researchers may not have found it necessary to include their operational definitions of resilience if this was not a focus of their study, limiting the number of definitions found. Grey literature, such as dissertations, case reports, and non-published studies, was excluded. By not examining sources outside of traditional publishing channels, the review may have missed important sources of information, which may have contributed to the understanding of resilience. Given the complexity of qualitative data, single author coding/analysis may lead to a loss of alternate interpretations of data, and thus potential themes may have been excluded, although we attempted to minimize this through collaborative discussion of the data set with other study authors.

Previous research has suggested that resilience is a developmental process unfolding over time and circumstances, with determinants of resilience differing depending on global, cultural, and contextually specific aspects and specific challenges ${ }^{(6)}$. As data was drawn from a global population of diverse sociocultural backgrounds with different cancer types and disease burdens, the relevance of our findings to specific cancer populations is limited. Additionally, the term "resilience" may not be used in non-English speaking backgrounds; nine of the included studies were from countries where English is not the official language, and another study explicitly sought participants who did not speak English as a first language. When translated into English, subtle cultural nuances of the term resilience may be lost.

\section{CONCLUSION}

This review found that patients are seldom quoted using the term resilience, and no cancer patient definitions of resilience were identified. Furthermore, usage and definitions of the term resilience by researchers are notably inconsistent. While patients do not use the term resilience, they identify various coping strategies, 
spirituality and growth, and social support as important attributes commonly associated with resilience. Further research specifically identifying how cancer patients understand and use the term "resilience" is needed.

\section{REFERENCES}

1. Luthar SS, Cicchetti D, Becker B. The Construct of Resilience: A Critical Evaluation and Guidelines for Future Work. 2000;71(3):543-62.

2. Min J, Yoon S, Lee C, Chae J, Lee C, Song K, et al. Psychological resilience contributes to low emotional distress in cancer patients. 2013;2469-76.

3. Eicher M, Matzka M, Dubey C, White K. Resilience in Adult Cancer Care: An Integrative Literature Review. 2015;42(1).

4. Bonanno GA, Westphal M, Mancini AD. Resilience to Loss and Potential Trauma. 2011;

5. Rosenberg A, Ma MS, Ms MB, Bs VK, Mph NE, Bs CW, et al. Paper Session ( TH320 e TH321) The ““ Promoting Resilience in Stress Management "' ( PRISM ) Intervention for Adolescents and Young Adults : A Pilot Randomized Controlled Trial ( TH320A ) Changes Over Time in Good-Parent Beliefs Among Parents of Children with Serious Illness: Two Year Cohort Study ( TH320B ) Family Satisfaction with Symptom Management Practices and Child Comfort During Pediatric Hematopoietic Stem Cell. J Pain Symptom Manage [Internet]. 2018;55(2):569-70. Available from: http://dx.doi.org/10.1016/j.jpainsymman.2017.12.023

6. Molina Y, Yi JC, Martinez-gutierrez J, Reding KW. Resilience Among Patients Across the Cancer Continuum: 2012;18(1).

7. Tong A, Flemming K, Mcinnes E, Oliver S, Craig J. Enhancing transparency in reporting the synthesis of qualitative research : ENTREQ. 2012;(Figure 1):1-8.

8. Helene S, Haug K, Ph D, Danbolt LJ, Kvigne K, Ph D. Older people with incurable cancer: Existential meaning-making from a life-span perspective. 2016;79:20-32.

9. Jahn AL, Herman L. Research in Human Development Distress and Resilience After Cancer in Veterans. 2012;(October 2014):37-41.

10. Pentz M. Journal of Gerontological Resilience Among Older Adults with Cancer and the Importance of Social Support and Spirituality-Faith. (December 2014):37-41.

11. Stefanic N, Caputi P, Lane L, Iverson DC. European Journal of Oncology Nursing Exploring the nature of situational goal-based coping in early-stage breast cancer patients: A contextual approach. Eur J Oncol Nurs [Internet]. 2015; Available from: http://dx.doi.org/10.1016/j.ejon.2015.03.008

12. Engeli L, Hospital P, Moergeli H. Resilience in patients and spouses faced with malignant melanoma . A qualitative longitudinal study. 2016;

13. Article O. Cancer patients' experiences with and perceived outcomes of yoga : results from focus groups. 2013;1861-70.

14. Bache RA, Bhui KS, Dein S, Korszun A. African and Black Caribbean origin cancer survivors: a qualitative study of the narratives of causes, coping and care experiences. 2012;17(April).

15. Harper FWK, Nevedal A, Eggly S, Francis C, Schwartz K, Albrecht TL. "It ' s up to you and God ": understanding health behavior change in older African American survivors of colorectal cancer.

16. Spek N Van Der, Vos J, Uden-kraan CF Van, Breitbart W, Tollenaar RAEM, Cuijpers P, et al. Meaning Making in Cancer Survivors : A Focus Group Study. 2013;8(9):22-6.

17. Grant M, Mcmullen CK, Altschuler A, Mohler MJ, Hornbrook MC, Herrinton LJ, et al. Gender Differences in Quality of Life Among Long-Term Colorectal Cancer Survivors With Ostomies. 2011;58796.

18. Macartney JI, Broom A, Kirby E, Good P, Wootton J. On resilience and acceptance in the transition to palliative care at the end of life. 2014;1-17.

19. Radcliffe O. Patients ' constructions of disability in metastatic spinal cord compression. 2009;132-40.

20. Baker P, Psychologist TC, Section HP, Baker P, Beesley H, Fletcher I, et al. " Getting back to normal " or " a new type of normal "? A qualitative study of patients" responses to the existential threat of cancer. 2016;(November 2014):180-9.

21. Skeath P, Norris S, Katheria V, White J, Baker K, Handel D, et al. The Nature of Life-Transforming Changes Among Cancer Survivors. 2013;

22. Saraf S, Singh TB, Khurana S. Cervical Cancer Survivors : Meaning in Life. 2013;58(June):144-52.

23. Grunfeld EA, Drudge-coates L, Rixon L, Eaton E, Cooper AF. “ The Only Way I Know How to Live Is to Work": A Qualitative Study of Work Following Treatment for Prostate Cancer. 2013;32(1):75-82.

24. Lam WWT, Yoon SW, Sze WK, Ng AWY, Soong I, Kwong A, et al. Comparing the meanings of living with advanced breast cancer between women resilient to distress and women with persistent distress : a qualitative study. 2016; 
25. Paul LB, Pitagora D, Brown B, Tworecke A, Rubin L. Support needs and resources of sexual minority women with breast cancer. 2014;584(November 2013):578-84.

26. Parry C. Embracing Uncertainty: An Exploration of the Experiences of Childhood Cancer Survivors. 2015;13(1):227-46.

27. Kong H, Kong H, Palliative M, Manitoba CC, Kong H, Kong H, et al. Living and dying with dignity in Chinese society: perspectives of older palliative care patients in Hong Kong. 2013;455-61.

28. Southwick SM, Bonanno GA, Masten AS, Panter- C, Yehuda R, Southwick SM, et al. interdisciplinary perspectives. 2017;8198(February).

29. I RGT, Calhoun LG. The Posttraumatic Growth Inventory: Measuring the Positive Legacy of Trauma. 1996;9(3):455-71. 